$\mathrm{CPR}$ and linked in the same way as cases. Finally, individual information on SES, residential history, reproduction, prescribed medicine, and comorbidity has been added to each case and control. Finally, a JEM on potential carcinogens can be applied.

Results Results confirm increased risk for e.g. lung and bladder cancer among painters and for nasal cancer in wood dust exposed workers. On the other hand, farmers, gardeners and forestry workers had deficits for many cancers. Results on new significant associations will also be presented.

Conclusions These data confirms many well established associations between work and cancer, and demonstrates that many associations are not fully explained. The large number of cancers available for analysis provides the opportunity to evaluate possible occupational associations even with rare cancers.

\section{LONG-TERM EFFECTS OF EXPOSURES AT OAK RIDGE NATIONAL LABORATORY}

B Richardson, Wing, Keil, Wolf. University of North Carolina, Chapel Hill, United States of America

\subsection{6/oemed-2013-101717.250}

We examine mortality in a cohort of workers at the Oak Ridge National Laboratory (ORNL), a US Department of Energy research and development facility. The last mortality follow-up of this epidemiologically important cohort was conducted 18 years ago and included workers hired between 1943 and 1972. Analyses of these data revealed a positive association between occupational radiation dose and all cancer mortality under a 20year lag assumption $(1.73 \%$ increase in all cancer mortality per $10 \mathrm{mSv}$, standard error $=0.86$ ). We have expanded this cohort to include 22,834 workers hired between 1943 and 1985, and updated mortality follow-up through 2008, yielding almost three times the number deaths observed in the last mortality follow-up of an ORNL cohort. Extending follow-up of this cohort is important not just for statistical power, but also because many important questions in radiation research concern the long term effects of irradiation, particularly on malignant diseases. These workers were individually badge-monitored for external exposure to ionising radiation, allowing evaluation of the effects of protracted radiation exposures accrued at work. We compared the observed deaths to expectations based upon US mortality rates, and evaluated radiation dose-mortality associations. Findings include excess deaths due to cancer of the pleura $(\mathrm{SMR}=$ 12.09 95\%CI: 4.44, 26.32), cancer of the bladder (SMR $=1.89$ 95\%CI: 1.26, 2.71), and leukaemia (SMR $=1.33$ 95\%CI: 0.87, 1.93) among hourly-paid males, and excess deaths due to cancer of the bladder (SMR $=2.20$ 95\%CI: 1.20, 3.69) and leukaemia $(\mathrm{SMR}=1.64$ 95\%CI: 1.09, 2.36) among females. Further results will be presented.

\section{Session: 5. Muscoskeletal disorders}

\section{DIFFERENCES IN THE DETERMINANTS OF EXTENSIVE AS COMPARED WITH MORE LIMITED MUSCULOSKELETAL PAIN}

D Coggon, G Ntani, K T Palmer. University of Southampton, Southampton, United Kingdom

10.1136/oemed-2013-101717.251
Objectives To explore whether multi-site musculoskeletal pain differs from more localised musculoskeletal pain in its association with risk factors.

Methods As part of the CUPID study, standardised questionnaires were used to ascertain exposure to risk factors and the prevalence of pain for a day or longer during the past month at each of 10 anatomical sites. Analysis was based on 12,410 participants from 47 occupational groups (mostly nurses and office workers) in 18 countries. Associations with risk factors were assessed by Poisson regression and summarised by prevalence rate ratios (PRRs).

Results Extensive pain (i.e. at six or more of the 10 anatomical sites examined) was much more prevalent than would have been expected by chance coincidence. In comparison with limited pain (i.e. at 1-3 sites), extensive pain showed distinctive associations with demographic characteristics and a much stronger relation to somatising tendency (PRR 4.6, 95\% CI 3.5-6.1 v 1.3, 95\% CI 1.2-1.4) and reported heavy physical loading (PRR 5.0, 95\% CI 2.8-9.2 v 1.4, 95\% CI 1.2-1.6). It also varied differently between occupational groups. Thus, for example, nurses in Spain had the highest rate of limited pain $(64.3 \%)$, but one of the lowest rates of extensive pain (3.5\%). In contrast, the prevalence of extensive pain among office workers in Nicaragua was $14.0 \%$, whereas that of limited pain was only $40.4 \%$.

Conclusions Extensive musculoskeletal pain has different determinants from pain affecting fewer anatomical sites. Its prevalence varies substantially between occupations and countries, and in a different way from limited pain. In research on causes of pain at specific anatomical sites, it may be important to distinguish cases with pain only at the site of interest from those with pain also at multiple other sites.

\section{DISABLING MUSCULOSKELETAL PAIN IN WORKING POPULATIONS: IS IT THE JOB THE PERSON OR THE CULTURE?}

D Coggon, G Ntani, K T Palmer. University of Southampton, Southampton, United Kingdom

\subsection{6/oemed-2013-101717.252}

Objectives To compare the prevalence of disabling low back pain (DLBP) and disabling wrist/hand pain (DWHP) among groups of workers carrying out similar physical activities in different cultural environments, and to explore explanations for observed differences.

Methods Standardised questionnaires were used to ascertain pain that interfered with everyday activities and exposure to possible risk factors in 12,426 participants from 47 occupational groups (mostly nurses and office workers) in 18 countries. Associations with risk factors were assessed by Poisson regression.

Results The one-month prevalence of DLBP in nurses varied between countries from $9.6 \%$ to $42.6 \%$, and that of DWHP in office workers from $2.2 \%$ to $31.6 \%$. Rates of disabling pain at the two anatomical sites co-varied $(r=0.76)$, but DLBP tended to be relatively more common in nurses and DWHP in office workers. Established risk factors such as occupational physical activities, psychosocial aspects of work and tendency to somatise were confirmed, and associations were found also with adverse health beliefs and group awareness of people outside work with musculoskeletal pain. However, after allowance for these risk factors, up to eightfold differences in prevalence remained. Systems of compensation for work-related illness, and financial 
support for health-related incapacity for work appeared to have little influence on the occurrence of symptoms.

Conclusions There is large international variation in the prevalence of disabling forearm and back pain among occupational groups carrying out similar tasks. This is only partially explained by the personal and socio-economic risk factors that were analysed.

\section{OCCUPATIONAL AND NON-OCCUPATIONAL RISK FACTORS FOR SURGICALLY TREATED CARPAL TUNNEL SYNDROME: PRELIMINARY RESULTS OF A MULTICENTRE POPULATION-BASED CASE-CONTROL STUDY}

${ }^{1} S$ C Curti, ${ }^{1}$ Mattioli, ${ }^{2}$ Baldasseroni, ${ }^{3}$ Bovenzi, ${ }^{1}$ Bonfiglioli, ${ }^{1}$ Violante. ${ }^{1}$ University of Bologna, Bologna, Italy; ${ }^{2}$ Tuscany Regional Centre for Occupational Injuries and Diseases (CeRIMP), Florence, Italy; ${ }^{3}$ University of Trieste, Trieste, Italy

\subsection{6/oemed-2013-101717.253}

Objectives Carpal tunnel syndrome (CTS) is a socially relevant condition that often involves disability. Few large analytical studies have been published considering occupational/non-occupational risk factors. This population-based case-control study aims at investigating both occupational and non-occupational risk factors for surgically treated CTS.

Methods Sixteen centres participated in the study. Each centre identified 200 subjects (aged 25-59 yr): 100 cases (50 women and 50 men) and 100 controls (50 women and 50 men). Cases were randomly drawn from administrative databases of all citizens submitted during 2001 to carpal tunnel release in any public/private hospital with a principal diagnosis of CTS. Controls were randomly sampled from official national health service registry records and were frequency matched by age and gender with cases. Participants were invited by mail (and phone when necessary) to respond to a structured questionnaire regarding occupational (including previous and current job titles along with biomechanical risk factors) and non-occupational risk factors.

Results The sixteen centres overall identified 3,052 subjects (1,458 cases and 1,594 controls) on the base of the study protocol criteria. A total number of 2,294 subjects responded to the questionnaire (1,182 cases and 1,112 controls) corresponding to a response rate of $81 \%$ and $70 \%$ respectively. After exclusion of non-eligible subjects, 1,018 cases and 959 controls entered the main analysis. After adjusting for non-occupational risk factors, manual workers of both sexes appeared to have at least 4-fold risk of surgical treatment of CTS, as compared with non-manual counterparts (women: OR 4.57, 95\% CI 3.07-6.81; men: OR 4.44; 95\% CI 2.96-6.67).

Conclusions This multicentre population-based case-control study strongly underscores the relevance of manual work as an important risk factor for surgically treated CTS, irrespectively of gender. Further analyses will contribute to provide conclusive evidence on the relationship between biomechanical overload and CTS, considering non-occupational risk factors as well.

\section{PSYCHOLOGICAL AND CULTURALLY INFLUENCED RISK FACTORS FOR THE INCIDENCE AND PERSISTENCE OF NON-DISABLING AND DISABLING MUSCULOSKELETAL PAIN. SPANISH CUPID STUDY}

'S V P Vargas-Prada, 'Serra, 'Martínez, ${ }^{2}$ Delclos, ${ }^{3}$ Coggon, 'Benavides. 'Center for Research in Occupational Health (CISAL). Universitat Pompeu Fabra, Barcelona, Spain; ${ }^{2}$ School of Public Health, University of Texas, Houston, United States of America; ${ }^{3} M R C$ Lifecourse Epidemiology Unit, University of Southampton, Southampton, United Kingdom

\subsection{6/oemed-2013-101717.254}

Objective To investigate the role of psychological risk factors for the development and persistence of non-disabling (NDMSP) and disabling (DMSP) musculoskeletal pain (MSP).

Methods As part of the CUPID study, 1105 nurses and office workers were asked at baseline about psychological and workrelated psychosocial risk factors, physical activities in the workplace and MSP in the past month and past year at ten anatomical sites (back, neck, and left and right shoulder, elbow, wrist/ hand and knee). One year later, pain in the past month was again ascertained. Pain was defined as disabling if certain daily activities were difficult or impossible to perform. At baseline, pain-free anatomical sites were included in the analyses for new NDMSP and DMSP, and painful sites in the analysis for persistent NDMSP and DMSP. Analysis was based on anatomical sites and associations were explored using multilevel multinomial logistic regression modelling.

Results 971 participants (87.9\%) completed follow-up. Among 8083 pain-free anatomical sites at baseline, 341 (4.2\%) and 412 (5.1\%) were involved in new NDMSP and DMSP at follow-up. After adjustment for sex, age, occupational risk factors and pain at the same site in the past year, new DMSP was predicted by somatising tendency (OR 3.4, 95\%CI 2.1-5.5). Among 1627 painful sites at baseline, $379(23.3 \%)$ and 500 (30.7\%) still had NDMSP and DMSP one-year later. Having adverse beliefs about the prognosis of MSP was associated with an increased risk of persistence of NDMSP (OR 2.3; 95\%CI 1.2-4.6) and DMSP (OR 2.9; 95\%CI 1.5-5.6).

Conclusion Psychological risk factors may have a stronger role in the development and persistence of DMSP than of NDMSP.

\section{MUSCULOSKELETAL DISORDERS AND PSYCHOLOGICAL COMORBIDITY IN GULF WAR VETERANS AND THE RELATIONSHIP WITH PHYSICAL AND MENTAL HEALTH AND WELLBEING}

L Kelsall, McKenzie, Roberts, Forbes, Urquhart, Sim. Monash University, Melburne, Australia

\subsection{6/oemed-2013-101717.255}

Objective To investigate musculoskeletal disorders, psychological comorbidity, and general physical and mental health and wellbeing in Gulf War veterans and a military comparison group.

Methods Cross-sectional study of 1456 male Australian 1990 1991 Gulf War veterans (veterans) and a non-Gulf comparison group ( $\mathrm{n}=1588$ ). At a medical assessment in 2000-2002, reported doctor diagnosed arthritis or rheumatism, back or neck problems, joint problems, soft tissue disorders were rated by medical practitioners as non-medical, unlikely, possible, or probable diagnoses. Only musculoskeletal disorders rated as probable diagnoses were included in analyses. DSM-IV psychological disorders were measured using the Composite International Diagnostic Interview. Physical and mental health and wellbeing was assessed using the 12-item Short-Form Health Survey (SF-12).

Results Almost one-quarter of veterans (24.5\%) and comparison group $(22.4 \%)$ reported a musculoskeletal disorder (odds ratio OR 1.19 ; 95\% CI 1.00-1.43). Having any or a specific musculoskeletal disorder was associated with depression and 